\title{
THE NEW TRAVELLING WAVE SOLUTIONS OF CONFORMABLE PARTIAL DIFFERENTIAL EQUATIONS
}

\section{ORKUN TASBOZAN, ALI KURT*}

Department of Mathematics, Hatay Mustafa Kemal University, Turkey

Corresponding author: pau.dr.alikurt@gmail.com

Received Nov. 26, 2018

\begin{abstract}
Aвstract. In this paper, the sine-Gordon expansion method is employed to have the new exact solutions of time fractional complex coupled Higgs field equation and combined Kdv-mKdv equations. By using some wave transformations and by the practicalness of chain rule and applicability of the conformable fractional derivative, the fractional partial differential equation turns into a nonlinear ordinary differential equation.
\end{abstract}

2010 Mathematics Subject Classification. 35R11, 35A20, 35C05.

Key words and phrases. sine-Gordon expansion method; coupled Higgs equation; Kdv-Mkdv equation; conformable fractional derivative.

\section{InTRODUCTION}

Until very recently, the problem of taking non-integer order of derivative or integration was quite hard for all disciplines of the science. However by development of mathematics knowledge, this problem came to a surmountable state. Although there are a number of different type of definition of fractional derivatives or integrations, Riemann-Liouville and Caputo are the most popular ones among them [1-3]. Despite the popularity of these derivatives, they do not provide some basic properties of integer order derivatives such as product rule, quotient rule, chain rule and etc. [4]. Recently a simple, well behaved and the limit based derivative that obeys the basic which obeys basic classical properties called conformable derivative is expressed by Khalil et. al. [4]

Definition 1.1. Let $f:[0, \infty) \rightarrow \mathbb{R}$ be a function. The $\alpha^{\text {th }}$ order "conformable fractional derivative" of $f$ is defined by,

$$
T_{\alpha}(f)(t)=\lim _{\varepsilon \rightarrow 0} \frac{f\left(t+\varepsilon t^{1-\alpha}\right)-f(t)}{\varepsilon}
$$

for all $t>0, \alpha \in(0,1)$.

Definition 1.2. If $f$ is $\alpha$-differentiable in some $(0, a), a>0$ and $\lim _{t \rightarrow 0^{+}} f^{(\alpha)}(t)$ exists then define $f^{(\alpha)}(0)=\lim _{t \rightarrow 0^{+}} f^{(\alpha)}(t)$. The "conformable fractional integral" of a function $f$ starting from $a \geq 0$ is defined as:

$$
I_{\alpha}^{a}(f)(t)=\int_{a}^{t} \frac{f(x)}{x^{1-\alpha}} d x
$$

DOI: 10.28924/APJM/6-8 
where the integral is the usual Riemann improper integral, and $\alpha \in(0,1]$.

Some properties of conformable derivative are indicated in the works $[4,5]$. After the appearance of conformable derivative, it aroused great interest by many scientists, and many applications of this derivative can be seen in the literary [6-11]. Avcr et. al. [12] find the fundamental solution of a conformable heat equation acting on a radial symmetric plate. Tariq et. al. [13] obtained the exact solution of Cahn-Allen equation by Tanh method via new conformable derivative. Hosseini et. al. [14] employed the Modified Kudryashov method for solving the conformable time fractional Klein-Gordon equations. In this work an application of the proposed method to a complex coupled equation and a nonlinear differential equation is illustrated. Fractional Higgs field equation

$$
\begin{aligned}
& D_{t}^{\alpha} D_{t}^{\alpha} u-u_{x x}-a u+b u|u|^{2}-2 u v=0, \\
& D_{t}^{\alpha} D_{t}^{\alpha} v+v_{x x}-b\left(|u|^{2}\right)_{x x}=0
\end{aligned}
$$

which describes a system of conserved scalar nucleons interacting with neutral scalar mesons. In this equation system, real constant $v$ shows a complex scalar nucleon field and $u$ a real scalar meson field. (1.1) represents the coupled nonlinear Klein-Gordon equation for $a<0, b<0$ and the coupled Higgs field equation for $a \geq 0, b>0$. Here we choose $a=0, b=1$.

The $\mathrm{KdV}$ and $\mathrm{mKdV}$ equations are most popular soliton equations and have been extensively examined. Though nonlinear terms of $\mathrm{KdV}$ and $\mathrm{mKdV}$ equations often simultaneously exist in some problems such as fluid physics and quantum field theory and form the combined KdV-mKdV equation. Conformable time fractional combined $\mathrm{KdV}$-mKdV equation can be written as

$$
D_{t}^{\alpha} u+p u u_{x}+q u^{2} u_{x}+\delta u_{x x x}=0
$$

where $\alpha \in(0,1)$ and $D_{t}^{\alpha} u$ means conformable fractional derivative of function $u(x, t)$.

\section{SINE-GORDON EXPANSION METHOD}

In this part, we demonstrate the study of sine-Gordon expansion method over the nonlinear PDEs. This method depends on the sine-Gordon [15] equation and wave transform [16]. Consider the sine-Gordon equation [17] which is presented as a model field theory;

$$
u_{x x}-u_{t t}=\tau^{2} \sin (u)
$$

where $u=u(x, t)$ and $\tau$ is a real constant. Regarding the wave transformation $u=u(x, t)=U(\xi), \xi=\mu(x-c t)$ over the Eqn. (2.1),we have the following nonlinear differential equation,

$$
U^{\prime \prime}=\frac{\tau^{2}}{\mu^{2}\left(1-c^{2}\right)} \sin (U)
$$

By full simplifying the Eq. (2.2), equation reduces to

$$
\left[\left(\frac{U}{2}\right)^{\prime}\right]^{2}=\frac{\tau^{2}}{\mu^{2}\left(1-c^{2}\right)} \sin ^{2}\left(\frac{U}{2}\right)+K,
$$


where $K$ is integration costant. Assuming $K=0, \Phi(\xi)=\frac{U}{2}, \varrho^{2}=\frac{\tau^{2}}{\mu^{2}\left(1-c^{2}\right)}$ and substituting into Eqn. (2.3), we obtain,

$$
\Phi^{\prime}=\varrho \sin (\Phi)
$$

regarding $\varrho=1$ in Eqn. (2.4), leads to

$$
\Phi^{\prime}=\sin (\Phi)
$$

Revealing the solution of (2.5) by separation of variables method, we acquire the following equations,

$$
\begin{aligned}
& \sin (\Phi)=\sin (\Phi(\xi))=\left.\frac{2 \zeta e^{\xi}}{\zeta^{2} e^{2 \xi}+1}\right|_{\zeta=1}=\operatorname{sech}(\xi), \\
& \cos (\Phi)=\sin (\Phi(\xi))=\left.\frac{\zeta^{2} e^{2 \xi}-1}{\zeta^{2} e^{2 \xi}+1}\right|_{\zeta=1}=\tanh (\xi)
\end{aligned}
$$

where $\zeta$ is integration constant. To obtain the solution of following nonlinear conformable PDE;

$$
G\left(u, D_{t}^{\alpha} u, D_{x} u, D_{x x} u, D_{t}^{\alpha} D_{t}^{\alpha} u, \ldots\right),
$$

we ruminate over,

$$
U(\xi)=\sum_{i=1}^{n} \tanh ^{i-1}(\xi)\left[B_{i} \operatorname{sech}(\xi)+A_{i} \tanh (\xi)\right]+A_{0},
$$

according to Eqns. (2.6) and (2.7), Eqn. (2.9) can be recomposed as

$$
U(\Phi)=\sum_{i=1}^{n} \cos ^{i-1}(\Phi)\left[B_{i} \sin (\Phi)+A_{i} \cos (\Phi)\right]+A_{0} .
$$

In the Eqn.(2.8) balancing the degrees between the highest order linear term and nonlinear term $n$ can be determined. Let the all the coefficients of $\cos ^{i}(\Phi)$ and $\sin ^{i}(\Phi)$ to be zero, it leads to an equation system. Solving system using an computer software such as Maple the values of $A_{i}, B_{i}, \mu$ and $c$ can be derived. Lastly subrogating the values of $A_{i}, B_{i}, \mu$ and $c$ in Eqn. (2.9), the traveling wave solutions can be obtained.

\section{Application of the Considered Method}

3.1. Analytical Solutions for Coupled Higgs Equation. Let start with time fractional coupled Higgs equation (1.1) where the derivatives are in conformable sense. Describing the wave variables,

$$
u(x, t)=e^{i \Theta} U(\xi), v(x, t)=V \xi, \Theta=p x+r \frac{t^{\alpha}}{\alpha}, \xi=x+w \frac{t^{\alpha}}{\alpha},
$$

where $0<\alpha \leq 1$. By using the chain rule [5] and substituting the varibles (3.1) into Eqn. system (1.1), we have

$$
\begin{aligned}
& \left(w^{2}-1\right) U^{\prime \prime}+\left(p^{2}-r^{2}\right) U-2 U V+U^{3}=0, \\
& \left(w^{2}+1\right) V^{\prime \prime}-2 U U^{\prime \prime}+2\left(U^{\prime}\right)^{2}=0 .
\end{aligned}
$$


Integrating the second equation in the system and neglecting the constant of integration we find,

$$
\left(w^{2}+1\right) V=U^{2} .
$$

Substituting Eqn. (3.3) into the first equation of the system, we find

$$
\left(w^{4}-1\right) U^{\prime \prime}+\left(w^{2}+1\right)\left(p^{2}-r^{2}\right) U+\left(w^{2}-1\right) U^{3}=0,
$$

where prime means differentiation with respect to $\xi$. Balancing terms $U^{\prime \prime}$ with $U^{3}$ and using (2.10) we obtain,

$$
3 n=n+2,
$$

so that

$$
n=1 .
$$

Using $n=1$ coupled with Eqn. (2.10) generates,

$$
U=B \sin (\xi)+A \cos (\xi)+C,
$$

and

$$
U^{\prime \prime}=-B(\sin (\xi))^{3}+B(\cos (\xi))^{2} \sin (\xi)-2 A(\sin (\xi))^{2} \cos (\xi)
$$

Substituting the Eqns. (3.5) and (3.6) into Eqn. (3.4) and setting all the coefficients of $\cos ^{i}(\xi)$ and $\sin ^{i}(\xi)$ to be zero, we obtain following equation system

$$
\begin{aligned}
& w^{2} A^{3}-A^{3}+2 w^{4} A-3 w^{2} B^{2} A+3 B^{2} A-2 A=0, \\
& -2 B+3 w^{2} B A^{2}+2 w^{4} B-3 B A^{2}-w^{2} B^{3}+B^{3}=0, \\
& -3 A^{2} c+3 w^{2} A^{2} c+3 B^{2} c-3 w^{2} B^{2} c=0 \\
& 6 w^{2} B A c-6 B A c=0 \\
& -r^{2} A+w^{2} p^{2} A-w^{2} r^{2} A-3 A c^{2}+3 w^{2} A c^{2}+p^{2} A-2 w^{4} A+3 w^{2} B^{2} A-3 B^{2} A+2 A=0, \\
& w^{2} p^{2} B+p^{2} B-w^{2} r^{2} B+3 w^{2} B c^{2}-r^{2} B-3 B c^{2}-w^{4} B+w^{2} B^{3}+B-B^{3}=0, \\
& w^{2} c^{3}-r^{2} c-c^{3}-w^{2} r^{2} c+p^{2} c+w^{2} p^{2} c-3 B^{2} c+3 w^{2} B^{2} c=0 .
\end{aligned}
$$

Solving the system with the aid of Maple 12, we get the following solution sets,

$$
p= \pm r, w= \pm 1
$$

By using these solutions, wave variables (3.1) and putting them into Eqn. (2.9) we obtain the $u(x, t)$. Then substituting $u(x, t)$ into Eqn. (3.3) we have the wave solutions of $v(x, t)$ as follows. 


$$
\begin{aligned}
& u_{1}(x, t)=\left(\cos \left(p x \pm \frac{p t^{\alpha}}{\alpha}\right)+i \sin \left(p x \pm \frac{p t^{\alpha}}{\alpha}\right)\right)\left(c+B \operatorname{sech}\left(x \pm \frac{t^{\alpha}}{\alpha}\right)+A \tanh \left(x \pm \frac{t^{\alpha}}{\alpha}\right)\right), \\
& v_{1}(x, t)=\frac{1}{2}\left(\cos \left(p x \pm \frac{p t^{\alpha}}{\alpha}\right)+i \sin \left(p x \pm \frac{p t^{\alpha}}{\alpha}\right)\right)^{2}\left(c+B \operatorname{sech}\left(x \pm \frac{t^{\alpha}}{\alpha}\right)+A \tanh \left(x \pm \frac{t^{\alpha}}{\alpha}\right)\right)^{2} .
\end{aligned}
$$

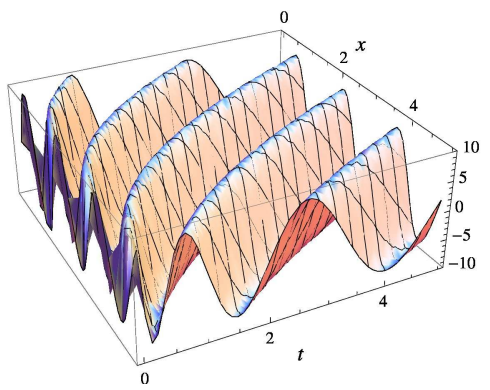

(A) Imaginary part of $v(x, t)$

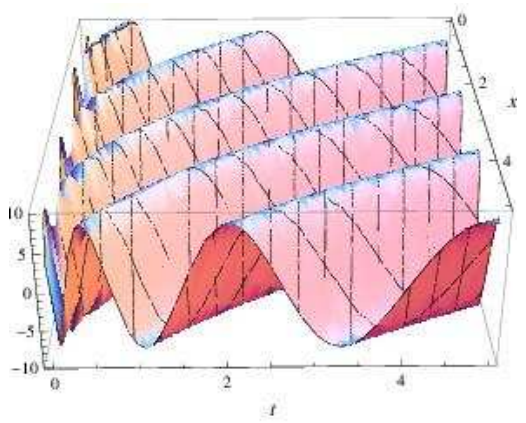

(в) Real part of $v(x, t)$

Figure 1. The physical behavior of imaginary and real parts of $v(x, t)$ for $A=2, B=2, c=2$, $p=2, \alpha=0.5$.

Now lets see the effect of $\alpha$ to the behavior of exact solution of $v(x, t)$

Let see the effect of $\alpha$ to the behavior of exact solution of $u(x, t)$

3.2. Analytical Solutions for Combined Kdv-mKdv Equation. In this subsection, we implement the SineGordon expansion method to solve the combined Kdv-mKdv equation (1.3). Applying the wave transform to the Eqn. (1.3)

$$
u(x, t)=U(\xi), \xi=m x+n \frac{t^{\alpha}}{\alpha},
$$

and integrating once yields the following equation

$$
n U+\frac{p m}{2} U^{2}+\frac{q m}{3} U^{3}+\delta m^{3} U^{\prime \prime}=0,
$$

where the prime denoted differentiation with respect to $\xi$. Balancing the degrees of highest order linear and nonlinear term gives us $n=1$. Now using (2.10), we can look for the solution of Eqn.(3.11) in the form

$$
U=B \sin (\xi)+A \cos (\xi)+C .
$$




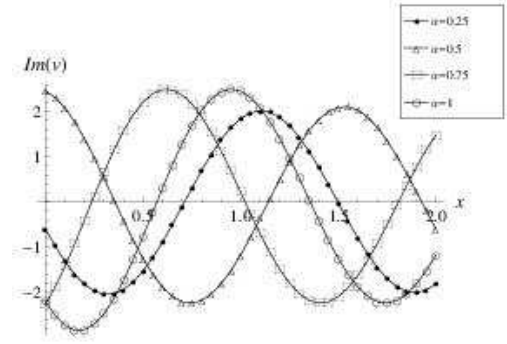

(A) Imaginary part of $v(x, t)$

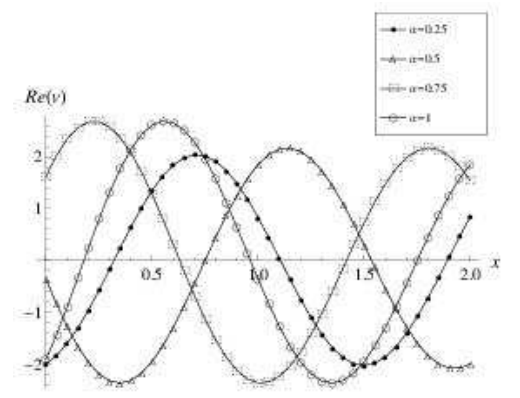

(в) Real part of $v(x, t)$

Figure 2. The effect of $\alpha$ to the physical behavior of imaginary and real parts of $v(x, t)$ for $A=1$, $B=1, c=1, p=2, t=2$.

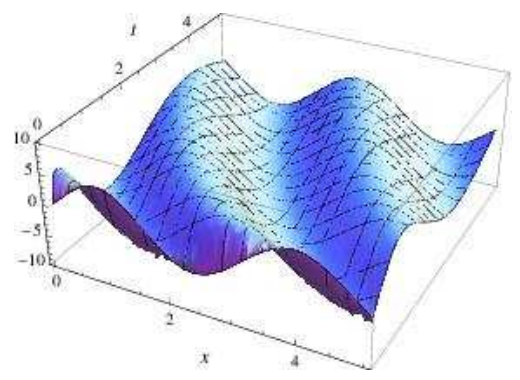

(A) Imaginary part of $u(x, t)$

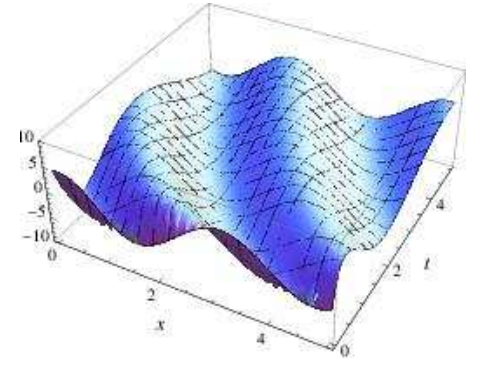

(в) Real part of $u(x, t)$

Figure 3. The physical behavior of imaginary and real parts of $v(x, t)$ for $A=2, B=2, c=2$, $p=2, \alpha=0.5$.

Considering Eqn.(3.12) we obtain

$$
U^{\prime \prime}=-B(\sin (\xi))^{3}+B(\cos (\xi))^{2} \sin (\xi)-2 A(\sin (\xi))^{2} \cos (\xi) .
$$




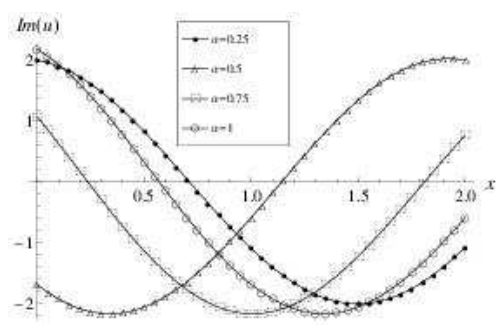

(A) $\operatorname{Im} u(x, t)$

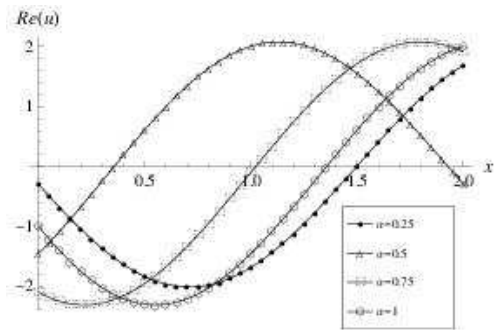

(в) $\operatorname{Re} u(x, t)$

FIGURE 4. The effect of $\alpha$ to the physical behavior of imaginary and real parts of $u(x, t)$ for $A=1$, $B=1, c=1, p=2, t=2$.

In that case using Eqns. (3.12) and (3.13), Eqn. (3.11) can be rewritten as follows

$$
\begin{aligned}
& n A \cos (\xi)+n B \sin (\xi)+n C+\frac{1}{2} p m A^{2}(\cos (\xi))^{2} \\
& +p m A \cos (\xi) B \sin (\xi)+p m A \cos (\xi) C+\frac{1}{2} p m B^{2}(\sin (\xi))^{2}+p m B \sin (\xi) C \\
& +\frac{1}{2} p m C^{2}+\frac{1}{3} q m A^{3}(\cos (\xi))^{3}+q m A^{2}(\cos (\xi))^{2} B \sin (\xi) \\
& +q m A^{2}(\cos (\xi))^{2} C+q m A \cos (\xi) B^{2}(\sin (\xi))^{2}+2 q m A \cos (\xi) B \sin (\xi) C+q m A \cos (\xi) C^{2} \\
& +\frac{1}{3} q m B^{3}(\sin (\xi))^{3}+q m B^{2}(\sin (\xi))^{2} C \\
& +q m B \sin (\xi) C^{2}+\frac{1}{3} q m C^{3}-\delta m^{3} B(\sin (\xi))^{3}+\delta m^{3} B(\cos (\xi))^{2} \sin (\xi) \\
& -2 \delta m^{3} A(\sin (\xi))^{2} \cos (\xi)=0 .
\end{aligned}
$$

Equating all the coefficients of $\cos ^{i}(\xi)$ and $\sin ^{i}(\xi)$ to be zero, we get the following equation system

$$
\begin{aligned}
& 1 / 3 q m A^{3}-q m B^{2} a+2 \delta m^{3} A=0, \\
& 1 / 2 p m A^{2}+q m A^{2} c-1 / 2 p m B^{2}-q m B^{2} C=0, \\
& q m B^{2} A-2 \delta m^{3} A+q m A C^{2}+p m A C+n A=0, \\
& 1 / 3 q m B^{3}+\delta m^{3} B+p m B C+q m B C^{2}+n B=0, \\
& 1 / 2 p m B^{2}+q m B^{2} C+1 / 2 p m C^{2}+1 / 3 q m C^{3}+n C=0, \\
& 2 q m B A C+p m b A=0, \\
& q m A^{2} B+2 \delta m^{3} B-1 / 3 q m B^{3}=0 .
\end{aligned}
$$


Using Maple 12 the solution of the above mentioned system, we have the following solution set

$$
\begin{aligned}
& \delta=-\frac{A^{2} q}{6 m^{2}}, n=-\frac{1}{3} A^{2} m q, p=0, B=0, C=0, \\
& A= \pm C, n=\frac{2}{3} C^{2} m q, p=-2 q C, \delta=-\frac{C^{2} q}{6 m^{2}} .
\end{aligned}
$$

Based on this solution set, we get the following solutions respectively,

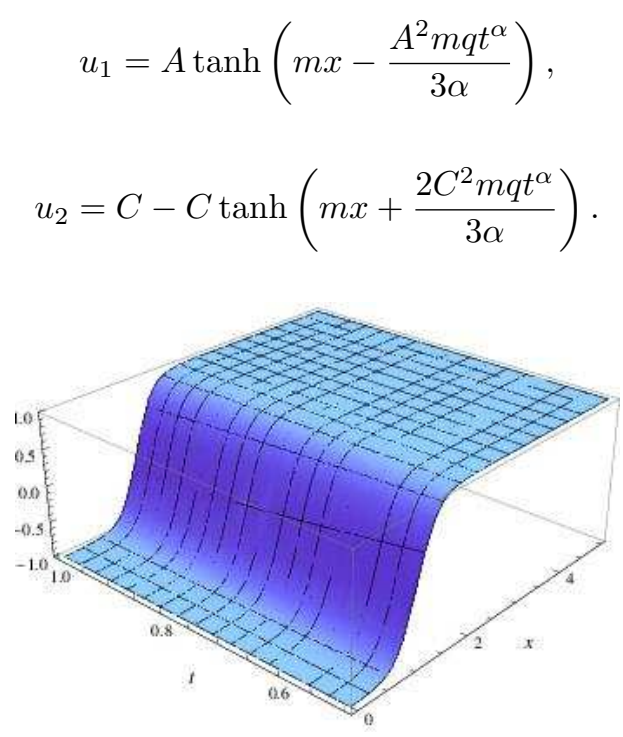

Figure 5. The physical behavior of $u(x, t)$ for $A=1, m=3, q=1, \alpha=0.25$.

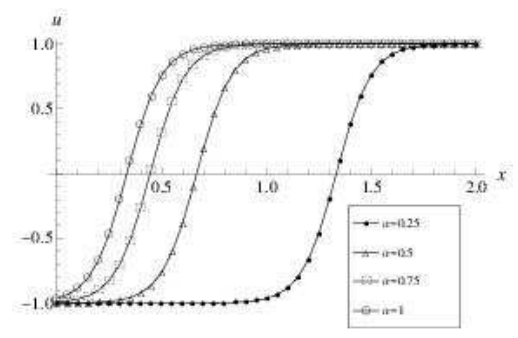

Figure 6. The effect of $\alpha$ to the physical behavior of $u(x, t)$ for $A=1, m=6, q=1, t=1$.

\section{Conclusions}

In this study, we investigated the wave behaviors of time fractional coupled Higgs equation and combined Kdv-mKdv equation by using sine-Gordon expansion method where fractional derivatives are in conformable sense. We obtained new hyperbolic function solutions both for the system (1.1) and the equation (1.3). All the solutions have been introduced with important physical information about the coupled Higss equation and combined Kdv-mKdv equation based on the results obtained along with figures (1-5). We have seen that the obtained solutions in this paper are absolutely solutions to equations (1.1) and (1.3). This is provided with the aid of Mathematica. Also 3-D graphics and comparative graphics for different values of $\alpha$ are given. Consequently we see that sine-Gordon expansion method can be easily applied to the fractional nonlinear PDEs with conformable 
derivative. Looking up to literary shows us none of the nonlinear PDEs with fractional derivative such as Caputo derivative or Riemann-Liouville derivative has analytical solution. Indeed conformable derivative is the unique derivative that we can obtain the exact solutions of nonlinear PDEs with fractional term by using it.

\section{REFERENCES}

[1] K.S. Miller, B. Ross, An Introduction to the Fractional Calculus and Fractional Differential Equations, John Wiley and Sons, New York, 1993.

[2] A.Kilbas, H.M. Srivastava, J.J. Trujillo, Theory and Applications of Fractional Differential Equations, Elsevier, San Diego, 2006.

[3] I. Podlubny, Fractional Differential Equations, Academic Press, San Diego, 1999.

[4] R. Khalil, M. Al Horani, A. Yousef, M. Sababheh, A new definition of fractional derivative, J. Comput. Appl. Math. 264 (2014), 65-70.

[5] T. Abdeljawad, On conformable fractional calculus, J. Comput. Appl. Math. 279 (2015), 57-66.

[6] A. Kurt, Y. Çenesiz, O. Tasbozan, On the solution of Burgers' equation with the new fractional derivative, Open Physics, 13(1) (2015), 355-360.

[7] O. Tasbozan, Y. Çenesiz, A. Kurt, New solutions for conformable fractional Boussinesq and combined KdV-mKdV equations using Jacobi elliptic function expansion method, Eur. Phys. J. Plus 131 (2016), 244.

[8] Y. Wang, J. Zhou, Y. Li, Fractional Sobolev's Spaces on Time Scales via Conformable Fractional Calculus and Their Application to a Fractional Differential Equation on Time Scales, Adv. Math. Phys. 2016(2016), 9636491.

[9] H. Karayer, D. Demirhan,F. Buyukkilic, Conformable Fractional Nikiforov-Uvarov Method, Commun. Theor. Phys. 66(1) (2016), 12.

[10] O.S. Iyiola, G. O. Ojo, On the analytical solution of Fornberg-Whitham equation with the new fractional derivative, Pramana J. Phys. 85(4) (2015), 567-575.

[11] A. Atangana, D. Baleanu, A. Alsaedi, New properties of conformable derivative, Open Physics, 13 (2015), 889.

[12] D. Avcı, B.B.I. Eroğlu, N. Özdemir, Conformable heat equation on a radial symmetric plate, Thermal Sci. 21(2) (2017), 819-826.

[13] H. Tariq, A. Ghazala, New traveling wave exact and approximate solutions for the nonlinear Cahn-Allen equation: evolution of a nonconserved quantity, Nonlinear Dyn. 88(1) (2017), 581-594.

[14] K. Hosseini, P. Mayeli, R. Ansari, Modified Kudryashov method for solving the conformable time-fractional Klein-Gordon equations with quadratic and cubic nonlinearities, Optik-Int. J. Light Electron Optics, 130 (2017), 737-742.

[15] C. Yan, A simple transformation for nonlinear waves, Phys. Lett. A, 224(1) (1996), 77.

[16] Y. Çenesiz, A. Kurt, New fractional complex transform for conformable fractional partial differential equations, J. Appl. Math. Stat. Inf. 12 (2016), 2.

[17] J. Rubinstein, Sine-Gordon Equation, J. Math. Phys. 11(1) (1970), 258-266. 